PROCEEDINGS OF THE

AMERICAN MATHEMATICAL SOCIETY

Volume 124, Number 10, October 1996

\title{
ON MAXIMAL FUNCTIONS IN ORLICZ SPACES
}

\author{
HIRO-O KITA
}

(Communicated by J. Marshall Ash)

\begin{abstract}
Let $\Phi(t)$ and $\Psi(t)$ be the functions having the representations $\Phi(t)=\int_{0}^{t} a(s) d s$ and $\Psi(t)=\int_{0}^{t} b(s) d s$, where $a(s)$ is a positive continuous function such that $\int_{1}^{\infty} \frac{a(s)}{s} d s=+\infty$ and $b(s)$ is quasi-increasing. Then the maximal function $M f$ is a function in Orlicz space $L^{\Phi}$ for all $f \in L^{\Psi}$ if and only if there exists a positive constant $c_{1}$ such that $\int_{1}^{s} \frac{a(t)}{t} d t \leq c_{1} b\left(c_{1} s\right)$ for all $s \geq 1$.
\end{abstract}

\section{INTRODUCTION}

Let $T$ be the group of real numbers modulo $2 \pi$, and let $f(x)$ be a real valued integrable function defined on $T$ with period $2 \pi$. The classical Hardy-Littlewood maximal function $M f(x)$ is defined by

$$
M f(x):=\sup _{x \in I} \frac{1}{|I|} \int_{I}|f(y)| d y,
$$

where the supremum is taken over all open intervals $I \subset T$ with $x \in I$.

The aim of this paper is to give a necessary and sufficient condition such that the Hardy-Littlewood maximal function $M f(x)$ defined by (1.1) is a function in Orlicz space $L^{\Phi}$, whenever $f(x)$ is an arbitrary function in Orlicz space $L^{\Psi}$. Orlicz space $L^{\Psi}$ is defined as follows.

Definition 1.1. Let $\Psi(t)$ be a nondecreasing continuous function such that $\lim _{t \rightarrow \infty} \Psi(t)=+\infty$. Put

$$
L^{\Psi}:=\left\{f: \int_{0}^{2 \pi} \Psi(\varepsilon|f(x)|) d x<+\infty \quad \text { for some } \quad \varepsilon>0\right\} .
$$

Then the space $L^{\Psi}$ is called an Orlicz space (see Kita and Yoneda [2], Rao and Ren [4] and Zygmund [6]).

We note that if $L^{\Psi}(t)=t^{p}$ for $t \geq 0$ and $p>1$, then $L^{\Psi}$ is a usual Lebesgue space.

Received by the editors December 6, 1993

1991 Mathematics Subject Classification. Primary 42B25, 46E30.

Key words and phrases. Hardy-Littlewood maximal function, Orlicz space.

(C)1996 American Mathematical Society 


\section{MAIN THEOREMS}

As is well known, the sublinear operator $M$ is of type $(p, p)(1<p \leq \infty)$ and weak type $(1,1)$ (see Torchinsky [5] and Zygmund [6]). For the maximal functions in the class $\Phi(L):=\left\{f: \int_{\mathbf{R}^{n}} \Phi(|f(x)|) d x<+\infty\right\}$, the detailed results can be found in the monograph of Kokilashvili and Krbec [3]. In this paper the maximal function of a function in Orlicz function space $L^{\Psi}$ defined by (1.2) will be considered.

A function $b(s)$ defined on $[0, \infty)$ is called quasi-increasing if there exists a positive constant $c_{0}$ such that $b\left(s_{1}\right) \leq c_{0} b\left(c_{0} s_{2}\right)$ for all $0<s_{1}<s_{2}$.

Let $a(s)$ and $b(s)$ be positive continuous functions defined on $[0, \infty)$ satisfying the following properties:

$$
b(s) \text { is quasi-increasing and } \lim _{s \rightarrow \infty} b(s)=+\infty .
$$

Put

$$
\Phi(t):=\int_{0}^{t} a(s) d s \text { and } \Psi:=\int_{0}^{t} b(s) d s \text { for } t \geq 0 .
$$

We have the following result which is also a generalization of the result in [5], p.103.

Theorem 2.1. Let $a(s), b(s), \Phi(t)$ and $\Psi(t)$ be the functions satisfying the above properties $(2.1)-(2.3)$. Then the following statements are equivalent:

(i) there exists a positive constant $c_{1}$ such that

$$
\int_{1}^{s} \frac{a(t)}{t} d t \leq c_{1} b\left(c_{1} s\right) \quad \text { for all } \quad s \geq 1
$$

(ii) there exists a positive constant $c_{2}$ such that

$$
\int_{0}^{2 \pi} \Phi(M f(x)) d x \leq c_{2}+c_{2} \int_{0}^{2 \pi} \Psi\left(c_{2}|f(x)|\right) d x \quad \text { for all } \quad f \in L^{1}(T) .
$$

As a corollary of Theorem 2.1 we have the following result.

Theorem 2.2. Let $a(s), b(s), \Phi(t)$ and $\Psi(t)$ be the functions satisfying $(2.1)-(2.3)$. Then the maximal function $M f$ is a function in Orlicz space $L^{\Phi}$ for all $f \in L^{\Psi}$ if and only if the functions $a(s)$ and $b(s)$ satisfy the inequality (2.4).

We note that in Theorems 2.1 and 2.2 the functions $\Phi(t)$ and $\Psi(t)$ do not necessarily satisfy the $\Delta_{2}$-condition, that is, there exist positive constants $c$ and $t_{0}$ such that $\Phi(2 t) \leq c \Phi(t)$ for all $t \geq t_{0}$.

Let us point out two particular consequences of the above theorems.

Corollary 2.3. Let $a(s)$ be a positive continuous function defined on $[0, \infty)$ such that $\int_{1}^{\infty} \frac{a(s)}{s} d s=+\infty$. Put $b(s):=\int_{1}^{s} \frac{a(t)}{t} d t+1$ for $s \geq 1, b(s)=1$ for $0 \leq s<1$, $\Phi(t):=\int_{0}^{t} a(s) d s$ and $\Psi(t):=\int_{0}^{t} b(s) d s$. Then for any Orlicz space $L^{\Psi_{1}} \nsubseteq L^{\Psi}$, there exists a function $f_{0} \in L^{\Psi_{1}}$ such that $f_{0} \notin L^{\Psi}$ and $M f_{0} \notin L^{\Phi}$.

Corollary 2.4. Let $a(s)$ be a positive quasi-increasing continuous function defined on $[0, \infty)$. Put $\Phi(t):=\int_{0}^{t} a(s) d s$. Then the following statements are equivalent: 
(i) there exists a positive constant $c_{1}$ such that

$$
\int_{1}^{s} \frac{a(t)}{t} d t \leq c_{1} a\left(c_{1} s\right) \quad \text { for all } \quad s \geq 1 .
$$

(ii) there exists a positive constant $c_{2}$ such that

$$
\int_{0}^{2 \pi} \Phi(M f(x)) d x \leq c_{2}+c_{2} \int_{0}^{2 \pi} \Phi\left(c_{2}|f(x)|\right) d x \quad \text { for all } f \in L^{1} .
$$

\section{Proof of the theorems}

First we prove Theorem 2.1. Let us prove (i) $\Rightarrow$ (ii). Let $\chi_{\{M f>1\}}(x)$ be a characteristic function on the set $\{x \in T: M f(x)>1\}$ and put $F(x):=$ $\Phi(M f(x)) \chi_{\{M f>1\}}(x)$. Then it follows that

$$
\begin{aligned}
I: & =\int_{M f>1} \Phi(M f(x)) d x=\int_{0}^{2 \pi} F(x) d x=\int_{0}^{\infty}|\{F>\lambda\}| d \lambda \\
& =\int_{0}^{\Phi(1)}|\{F>\lambda\}| d \lambda+\int_{\Phi(1)}^{\infty}|\{F>\lambda\}| d \lambda \\
& \leq 2 \pi \Phi(1)+\int_{\Phi(1)}^{\infty}|\{F>\lambda\}| d \lambda .
\end{aligned}
$$

From (2.1) and (2.3), $\Phi(t)$ is strictly increasing. Therefore we get

$$
I \leq 2 \pi \Phi(1)+\int_{\Phi(1)}^{\infty}\left|\left\{M f>\Phi^{-1}(\lambda)\right\}\right| d \lambda
$$

Put $t=\Phi^{-1}(\lambda)$. From (2.1) and (2.3) $\lim _{t \rightarrow \infty} \Phi(t)=+\infty$ holds. Therefore we have

$$
\int_{\Phi(1)}^{\infty}|\{F>\lambda\}| d \lambda=\int_{1}^{\infty}|\{M f>t\}| a(t) d t
$$

Since the sublinear operator $M$ is simultaneously of weak-type $(1,1)$ and of type $(\infty, \infty)$, it follows by the well known result (see Torchinsky [5], p.92) that there exist positive constants $c_{3}$ and $c_{4}$ such that

$$
|\{M f>t\}| \leq \frac{c_{3}}{t} \int_{t / c_{4}}^{\infty}|\{|f|>s\}| d s \quad \text { for all } \quad t>0 .
$$

Therefore it follows from (3.1), (3.2) and (3.3) that

$$
\begin{aligned}
I & \leq 2 \pi \Phi(1)+c_{3} \int_{1}^{\infty} \frac{a(t)}{t}\left(\int_{t / c_{4}}^{\infty}|\{|f|>s\}| d s\right) d t \\
& =2 \pi \Phi(1)+c_{3} \int_{1 / c_{4}}^{\infty}\left(|\{|f|>s\}| \int_{1}^{c_{4} s} \frac{a(t)}{t} d t\right) d s .
\end{aligned}
$$

By the assumption (i), we have

$$
\begin{aligned}
I & \leq 2 \pi \Phi(1)+c_{1} c_{3} \int_{1 / c_{4}}^{\infty}|\{|f|>s\}| b\left(c_{1} c_{4} s\right) d s \\
& \leq 2 \pi \Phi(1)+\left(c_{3} / c_{4}\right) \int_{0}^{2 \pi} \Psi\left(c_{1} c_{4}|f(x)|\right) d x .
\end{aligned}
$$

We conclude that (2.5) holds. 
Next we prove (ii) $\Rightarrow$ (i). Let (ii) hold and we assume that (i) does not hold. Then there exists a sequence of numbers $\left\{s_{k}: k \geq 1\right\}$ such that $s_{k} \geq 1$ for $k \geq 1$ and

$$
\int_{1}^{s_{k}} \frac{a(t)}{t} d t>2^{k} b\left(k 2^{k} s_{k}\right) \quad \text { for } \quad k \geq 1
$$

We choose a collection of disjoint open intervals $\left\{I_{k}: k \geq k_{0}\right\}, I_{k} \subseteq T$, such that

$$
\left|I_{k}\right|:=\frac{1}{2^{k} \Psi\left(2^{k} s_{k}\right)} \quad \text { for } \quad k \geq k_{0} \quad \text { and } \quad \sum_{k=k_{0}}^{\infty}\left|I_{k}\right|<2 \pi
$$

Put

$$
f(x):=\frac{\varepsilon_{0}}{c_{2}} \sum_{k=k_{0}}^{\infty} 2^{k} s_{k} \chi_{I_{k}}(x), \quad 0<\varepsilon_{0}<1
$$

where $\chi_{I_{k}}$ is a characteristic function on $I_{k}$ and a number $\varepsilon_{0}$ will be defined later. Then it follows from (3.5) and (3.6) that

$$
\begin{aligned}
\int_{0}^{2 \pi} \Psi\left(c_{2}|f(x)|\right) d x & =\sum_{k=k_{0}}^{\infty} \int_{I_{k}} \Psi\left(c_{2}|f(x)|\right) d x \\
& =\sum_{k=k_{0}}^{\infty} \Psi\left(\varepsilon_{0} 2^{k} s_{k}\right)\left|I_{k}\right| \leq \sum_{k=k_{0}}^{\infty} \Psi\left(2^{k} s_{k}\right) \cdot \frac{1}{2^{k} \Psi\left(2^{k} s_{k}\right)} \\
& =\sum_{k=k_{0}}^{\infty} 2^{-k}<+\infty
\end{aligned}
$$

Therefore we get $f \in L^{\Psi}$. Since $b(s)$ is quasi-increasing, we have

$$
\Psi(t) \geq \int_{t / 2}^{t} b(s) \geq \frac{1}{c_{0}} \int_{t / 2}^{t} b\left(\frac{t}{2 c_{0}}\right) d s=\frac{t}{2 c_{0}} b\left(\frac{t}{2 c_{0}}\right) .
$$

Therefore $L^{\Psi} \subseteq L^{1}$ holds and we can choose $\varepsilon_{0}$ such that

$$
|f|_{T}:=\frac{1}{2 \pi} \int_{0}^{2 \pi}|f(x)| d x<1
$$

On the other hand, for each $0<\varepsilon<1, \int_{0}^{2 \pi} \Phi(\varepsilon M f(x)) d x=+\infty$ holds. Indeed, put $g=\varepsilon f$. It is a well known fact (see Torchinsky [5], p.93) that there exists a positive constant $c_{5}$ such that

$$
|\{M g>\lambda\}| \geq \frac{c_{5}}{\lambda} \int_{|g|>\lambda}|g(x)| d x \quad \text { for all } \quad \lambda>|g|_{T} .
$$


Therefore it follows from (3.7) and (3.8) that

$$
\begin{aligned}
\int_{0}^{2 \pi} \Phi(\varepsilon M f(x)) d x & =\int_{0}^{2 \pi} \Phi(M g(x)) d x=\int_{0}^{\infty}|\{M g>\lambda\}| \Phi^{\prime}(\lambda) d \lambda \\
& \geq \int_{|g|_{T}}^{\infty}|\{M g>\lambda\}| \Phi^{\prime}(\lambda) d \lambda \geq \int_{1}^{\infty}|\{M g>\lambda\}| \Phi^{\prime}(\lambda) d \lambda \\
& \geq c_{5} \int_{1}^{\infty}\left(\int_{|g|>\lambda}|g(x)| d x\right) \frac{\Phi^{\prime}(\lambda)}{\lambda} d \lambda \\
& =c_{5} \int_{1}^{\infty}\left(\int_{0}^{2 \pi}|g(x)| \chi_{\{|g|>\lambda\}}(x) d x\right) \frac{\Phi^{\prime}(\lambda)}{\lambda} d \lambda \\
& =c_{5} \int_{0}^{2 \pi}|g(x)|\left(\int_{1}^{\infty} \chi_{\{|g|>\lambda\}}(x) \frac{\Phi^{\prime}(\lambda)}{\lambda} d \lambda\right) d x \\
& =c_{5} \int_{|g|>1}|g(x)|\left(\int_{1}^{|g(x)|} \frac{a(\lambda)}{\lambda} d \lambda\right) d x .
\end{aligned}
$$

Since $g(x)=\varepsilon f(x)$ and $f(x)$ is constant on $I_{k}$, we get

$$
g(x)=\frac{\varepsilon \varepsilon_{0}}{c_{2}} 2^{k} s_{k} \geq \frac{\varepsilon \varepsilon_{0} 2^{k}}{c_{2}} \uparrow+\infty \quad \text { as } \quad k \uparrow+\infty .
$$

Thus, there exists a positive integer $k_{1}>k_{0}$ such that

$$
\frac{\varepsilon \varepsilon_{0} 2^{k}}{c_{2}}>1 \quad \text { for all } \quad k \geq k_{1} .
$$

From (3.4), (3.5) and (3.9), it follows that

$$
\begin{aligned}
\int_{0}^{2 \pi} \Phi(\varepsilon M f(x)) d x & \geq c_{5} \sum_{k=k_{1}}^{\infty} \int_{I_{k}}|g(x)|\left(\int_{1}^{|g(x)|} \frac{a(\lambda)}{\lambda} d \lambda\right) d x \\
& =\frac{c_{5} \varepsilon \varepsilon_{0}}{c_{2}} \sum_{k=k_{1}}^{\infty} 2^{k} s_{k}\left(\int_{1}^{\frac{\varepsilon \varepsilon_{0} 2^{k} s_{k}}{c_{2}}} \frac{a(\lambda)}{\lambda} d \lambda\right)\left|I_{k}\right| \\
& \geq \frac{c_{5} \varepsilon \varepsilon_{0}}{c_{2}} \sum_{k=k_{1}}^{\infty} 2^{k} s_{k}\left(\int_{1}^{s_{k}} \frac{a(\lambda)}{\lambda} d \lambda\right) \cdot \frac{1}{2^{k} \Psi\left(2^{k} s_{k}\right)} \\
& \geq \frac{c_{5} \varepsilon \varepsilon_{0}}{c_{2}} \sum_{k=k_{1}}^{\infty} 2^{k} s_{k} \cdot 2^{k} s_{k} b\left(k 2^{k} s_{k}\right) \cdot \frac{1}{2^{k} \Psi\left(2^{k} s_{k}\right)} .
\end{aligned}
$$

Since $b(s)$ is quasi-increasing, it follows that

$$
\Psi\left(2^{k} s_{k}\right)=\int_{0}^{2^{k} s_{k}} b(s) d s \leq \int_{0}^{2^{k} s_{k}} c_{0} b\left(c_{0} 2^{k} s_{k}\right) d s=c_{0} 2^{k} s_{k} b\left(c_{0} 2^{k} s_{k}\right) .
$$

Therefore we have

$$
\begin{aligned}
\int_{0}^{2 \pi} \Phi(\varepsilon M f(x)) d x & \geq \frac{c_{5} \varepsilon \varepsilon_{0}}{c_{2}} \sum_{k=k_{1}}^{\infty} \frac{2^{k} s_{k} \cdot 2^{k} b\left(k 2^{k} s_{k}\right)}{2^{k} \cdot c_{0} 2^{k} s_{k} \cdot b\left(c_{0} 2^{k} s_{k}\right)} \\
& =\frac{c_{5} \varepsilon \varepsilon_{0}}{c_{2} c_{0}} \sum_{k=k_{1}}^{\infty} \frac{b\left(k 2^{k} s_{k}\right)}{b\left(c_{0} 2^{k} s_{k}\right)} .
\end{aligned}
$$


If we choose a positive integer $k_{2}>k_{1}$ such that $k_{2} \geq c_{0}^{2}$, then $b\left(c_{0} 2^{k} s_{k}\right) \leq$ $c_{0} b\left(c_{0} \cdot \frac{k}{c_{0}} 2^{k} s_{k}\right)$ for all $k \geq k_{2}$. Therefore we have

$$
\int_{0}^{2 \pi} \Phi(\varepsilon M f(x)) d x \geq \frac{c_{5} \varepsilon \varepsilon_{0}}{c_{2} c_{0}^{2}} \sum_{k=k_{2}}^{\infty} 1=+\infty .
$$

We arrive at a contradiction and Theorem 2.1 is proved.

Last we prove Theorem 2.2. Let us assume that the inequality (2.4) holds. Let $f$ be an arbitrary function in Orlicz space $L^{\Psi}$. Then there exists a positive number $\varepsilon_{1}$ such that $\int_{0}^{2 \pi} \Psi\left(\varepsilon_{1}|f(x)|\right) d x<+\infty$. By Theorem 2.1, we have the following inequality:

$$
\int_{0}^{2 \pi} \Phi\left(\frac{\varepsilon_{1}}{c_{2}} M f(x)\right) d x \leq c_{2}+c_{2} \int_{0}^{2 \pi} \Psi\left(\varepsilon_{1}|f(x)|\right) d x<+\infty .
$$

Thus $M f \in L^{\Phi}$ holds.

Conversely, let us assume that (2.4) does not hold. Then, by the same manner as the proof of Theorem 2.1, it follows that there exists a function $f \in L^{\Psi}$ such that $M f \notin L^{\Phi}$. This is a contradiction and the proof is complete.

Remark. Let $a(s)$ be a positive quasi-increasing continuous function defined on $[0, \infty)$. Put $\Phi(t)=\int_{1}^{t} a(s) d s$ for $t \geq 1$ and $\Phi(t)=0$ for $0 \leq t<1$. It is easy to see from the result given in [3] (see p.6, Theorem 1.2.1) that the following statements are equivalent:

(i) there exists a positive constant $c_{1}$ such that

$$
\int_{1}^{t} \frac{a(s)}{s} d s \leq c_{1} a\left(c_{1} t\right) \quad \text { for all } \quad t \geq 1
$$

(ii) there exists a positive constant $c_{2}$ such that

$$
\int_{1}^{t} \frac{\Phi(s)}{s^{2}} d s \leq \frac{c_{2} \Phi\left(c_{2} t\right)}{t} \quad \text { for all } \quad t \geq 1
$$

(iii) there exists a constant $\ell>1$ such that

$$
\Phi(t) \leq \frac{1}{2 \ell} \Phi(\ell t) \quad \text { for all } t \geq 1
$$

(iv) the function $\Phi^{\alpha}$ is quasi convex on $[0, \infty)$ for some $0<\alpha<1$, that is, there exist a convex function $\omega$ and a constant $c>0$ such that $\omega(t) \leq \Phi(t) \leq c \omega(c t)$ for all $t \geq 0$.

We note that if $a(t)$ is an increasing function on $[0, \infty)$ satisfying the inequality in the statement of (i), then there exist positive constants $\alpha, t_{0}$ and $c_{0}$ such that $a(t) \geq c_{0} t^{\alpha}$ for all $t \geq t_{0}$ (see Rao and Ren [4], p.26, Corollary 5).

\section{Examples}

In this section, some examples of functions $\Phi(t), \Psi(t), a(t)$ and $b(t)$ will be given. Let $\varphi_{1}(t)$ and $\varphi_{2}(t)$ be the functions defined on $[0, \infty)$. We write $\varphi_{1}(t) \sim \varphi_{2}(t)$, if there exist positive constants $c_{1}, c_{2}$ and $t_{0}$ such that $c_{1} \varphi_{1}(t) \leq \varphi_{2}(t) \leq c_{2} \varphi_{1}(t)$ for all $t \geq t_{0}$. 
Example 1. Let $1<p<+\infty$.

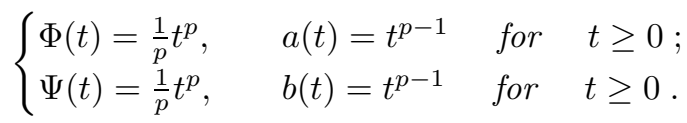

Example 2. Let $0<\alpha \leq 1$.

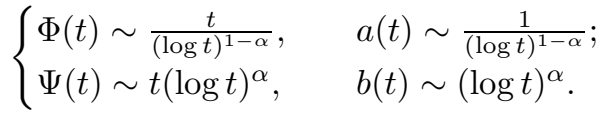

Example 3.

$$
\left\{\begin{aligned}
\Phi(t) & \sim \frac{t}{\log t}, & a(t) & \sim \frac{1}{\log t} \\
\Psi(t) & \sim t(\log \log t), & b(t) & \sim \log \log t .
\end{aligned}\right.
$$

Example 4. Put $\log ^{+} t=\log t$ for $t \geq 1$ and $\log ^{+} t=0$ for $0 \leq t<1$. And put $L_{1}(t)=\log ^{+} t, L_{n}(t)=\log ^{+} L_{n-1}(t)$ for $n \geq 2$.

$$
\left\{\begin{aligned}
\Phi(t) & \sim \frac{t}{L_{1}(t) L_{2}(t) \cdots L_{n-1}(t)}, & a(t) & \sim \frac{1}{L_{1}(t) L_{2}(t) \cdots L_{n-1}(t)} \\
\Psi(t) & \sim t L_{n}(t), & b(t) & \sim L_{n}(t) .
\end{aligned}\right.
$$

\section{REFERENCES}

1. M. de Guzmán, Differentiation of Integrals in $R^{n}$, Lecture Notes in Math., 481, SpringerVerlag, Berlin -Heidelberg-New York, 1975. MR 56:15866

2. H. Kita and K. Yoneda, A treatment of Orlicz spaces as a ranked space, Math. Japon. 37 (1992), 775-802. MR 93i:46054

3. V. Kokilashvili and M. Krbec, Weighted inequalities in Lorentz and Orlicz spaces, World Scientific, 1991. MR 93g:42013

4. M.M. Rao and Z.D. Ren, Theory of Orlicz Spaces, Marcel Dekker, 1991. MR 92e:46059

5. A. Torchinsky, Real variable methods in harmonic analysis, Academic Press, New York, 1986. MR 88e: 42001

6. A. Zygmund, Trigonometric series, Cambridge Univ. Press, Cambridge, 1959. MR 21:6498

Department of Mathematics, Faculty of Education, Oita University, 700 Dannoharu OITA 870-11, JAPAN

E-mail address: hkita@oita-cc.cc.oita-u.ac.jp 